\title{
HACIA UNA COMPRENSIÓN DEL CONFLICTO EN JUSTICIA ALTERNATIVA
}

\author{
TOWARDS AN UNDERSTANDING OF THE CONFLICT IN ALTERNATIVE JUSTICE
}

\author{
Francisca Silva-Hernández ${ }^{1} *$ \\ 1. Universidad Juárez Autónoma de Tabasco \\ División Académica de Ciencias Sociales y Humanidades, México \\ *Correspondencia del Autor: Francisca Silva Hernández, correo electrónico: fany987@hotmail.com
}

\section{RESUMEN}

El conflicto es un proceso de manifestación de pensamiento y conducta a partir de situaciones socioculturales en toda sociedad. Está visto como una situación compleja suscitada entre dos o más personas. Sin embargo, en justicia alternativa la perspectiva del conflicto tiene una comprensión, gestión y solución basada en el diálogo y comunicación entre las personas involucradas, lo que propicia protagonismo, empoderamiento y legitimación de las personas para resolver su propio conflicto. Este documento pretende abordar las generalidades del conflicto con el fin de afianzar una cultura de paz, convivencia y bienestar entre los sujetos inmersos en una sociedad en el que la gestión y solución del conflicto sea aplicado de forma constructiva y colaborativa para la construcción de ciudadanos capaces de realizar la transición de una mejor sociedad.

Palabras clave : Conflicto; cultura de paz; derechos humanos; mecanismos alternativos; sana convivencia.

Cómo citar:

Silva-Hernández, Francisca. (2020). Hacia una comprensión del conflicto en justicia alternativa. Revista de Investigaciones Universidad del Quindio, 32(1), 61-65. https://doi.org/10.33975/riuq.vol32n1.435

Información del artículo: Recibido: 09 marzo 2020; Aceptado: 15 marzo 2020 


\begin{abstract}
Conflict is a process of manifestation of thought and behavior from sociocultural situations in every society. It is seen as a complex situation between two or more people. However, in alternative justice, the perspective of the conflict has an understanding, management and solution based on dialogue and communication between the people involved, which encourages protagonism, empowerment and legitimacy of the people to resolve their own conflict. This document aims to address the generalities of the conflict in order to consolidate a culture of peace, coexistence and well-being among the subjects immersed in a society in which the management and solution of the conflict is applied in a constructive and collaborative way for the construction of capable citizens to make the transition to a better society.
\end{abstract}

Keywords: Conflict; culture of peace; human rights; alternative mechanisms; healthy coexistence.

\section{INTRODUCCIÓN}

Hacia una comprensión del conflicto en justicia alternativa, analiza de manera descriptiva la conceptualización del conflicto como acción que deriva de la consecuencia de relaciones sociales entre dos o más personas que lleva implícito percepciones, formas de comunicación e interpretación de hechos o sucesos que parten de actos simples hasta procesos complejos que requieren la asistencia de una gestión y solución del conflicto de manera profesional.

Se abordan diversos autores del conflicto que manifiestan la importancia de la comunicación, diálogo y escucha activa que debe existir al momento en que se aborda ya que, en caso de omitirlo, existe mayor probabilidad de obtener una mala gestión y por tanto una no favorable solución del mismo, resultando como usualmente acontece una repartición de lo que se está en disputa.

En la justicia alternativa el conflicto representa una vía pacífica de empoderamiento y legitimación de las personas para resolver sus propios conflictos. Para ello, los mecanismos alternativos de solución de controversias en la justicia alternativa representan un proceso de transición de los cuales se deben señalar los elementos que lo constituyen. Sin embargo, así como tiene sus particularidades también llega a tener sus genera- lidades, en el sentido que se pueden aproximar algunos con características y elementos parecidos, lo que permite dar pauta a abordar conflictos de una manera similar teniendo una adaptación en tiempo, modo y lugar de acuerdo al contexto o medio.

Finalmente, se aborda una visión del conflicto (Silva, 2008) distinta a la que se tiene, es decir del tradicional pensamiento negativo a uno positivo que permite la transformación de acuerdo a como se aborda desde el acceso a la justicia alternativa; siendo esto un proceso de cambio gradual y evolutivo en la propia sociedad ya que se visualiza a una cultura de paz y concordia a partir de mecanismos o estrategias que consienten a una convivencia de diálogo, democrática, de consenso, de poder sentarse, hablar y escuchar activamente a todas las partes que estén involucradas en el conflicto.

\section{METODOLOGÍA}

El conflicto en la justicia alternativa ayuda a crear una cultura de corresponsabilidad, respeto y tolerancia, la interacción y dinámica de la asociación de las relaciones que entablan los individuos pueden ser espontánea o voluntaria, en la que diversas razones (territoriales, culturales, sociales, temporalidad de la relación, objetivos comunes u otros) establecen una relación de dependencia 
recíproca entre sus miembros.

La revisión de material bibliohemerográfico presta a emplear una metodología cualitativa vista como una forma multidisciplinar de acercarse al conocimiento de la realidad social (Ibañez, 2002), de método documental que permitió vislumbrar la estrategia de comprensión y análisis de realidades teóricas o empíricas, basada en un enfoque descriptivo, analítico; permitiendo identificar a partir de la consulta de bases de datos, artículos científicos y obras de libro o capítulos de libro las generalidades del conflicto visto desde la justicia alternativa.

\section{RESULTADOS}

\section{Justicia alternativa}

La justicia alternativa se puede entender según Mondragón (2012) como una estructura procesal distinta de la jurisdiccional para la solución de controversias entre particulares. En el marco de la misma se enuncian mecanismos como la negociación, mediación, conciliación y arbitraje que establecen mediante procesos económicos en tiempo y dinero.

Nava y Breceda (2017) describen que los medios alternativos de solución de conflictos constituyen mecanismos convencionales, expeditos y económicos de solución de controversias; incluyen: i) los sistemas de negociación que buscan crear un ambiente que permitan a las partes alcanzar una solución razonable por sí mismos; ii) se extienden a los sistemas que cuentan con la intervención de un tercero ajeno a la disputa, que auxiliando (mediación) o proponiendo (conciliación) coopera para que éstas lleguen a un acuerdo por ellas mismas, y iii) alcanza a las modalidades adversariales a través de las cuales el tercero decide o resuelve (arbitraje).

En el estado mexicano el acceso a la justicia mediante los mecanismos alternativos antepone procesos favorecedores para el sistema de justicia en México, debido a que coadyuva al sistema de justicia heterocompositivo.

En ese sentido, el derecho de acceder a la justicia no es sólo la entrada de la persona o la admisión de una petición, es proteger el derecho a la verdad, la justicia y la reparación, asumir responsabilidades (Castillo y Bautista, 2018).

La justicia alternativa encamina a los ciudadanos a un abanico de posibles soluciones, ha procesos conducidos al reconocimiento de formas de autonomía por una parte y auto regulación por otra, que estarían generando auténticos sistemas plurales de fuentes judiciales que en muchos casos se caracterizan por un marcado carácter informal (Calvo García, 2009).

Además de ver la justicia alternativa desde el ámbito jurídico en el cambio transicional de las diversas sociedades se está adoptando como un mecanismo que coadyuve a las sociedades con ciudadanos en toma de decisiones por consenso para un bien colectivo. Actuando y enseñando las ideas e ideales de la resolución de conflictos manejados a través de las alternativas de resolución entre quienes conforman la sociedad, contribuyendo a la reducción de la violencia y al fortalecimiento de espacios pacíficos para las futuras generaciones (Fuquen 2003).

Finalmente, la justicia alternativa rebasa el abordar conflictos de índole judicial, debido a que abona a conflictos de índole comunitaria, educativa, ambientales, entre otros. Lo que ha implicado en acciones de políticas públicas como integrar o incorporar a programas educativos los mecanismos alternativos en pro de una sociedad pacífica abierta al diálogo y comunicación.

\section{EI conflicto}

La justicia alternativa a través de los mecanismos alternativos de solución de controversias configura nuevos procesos de apropiación y comprensión del conflicto.

El conflicto regularmente se asocia a una idea o 
pensamiento de problemas, disputa, situaciones de enfrentamiento entre dos o más personas en que uno sale con mayor o absoluta ventaja en comparación del otro; se entiende también en un sentido adversarial que conlleva a un escenario de enojos, malas intenciones, mentiras, dolor, sentimientos (Alcaraz \& Lorenzo, 2016) que impulsan a plantearse en una posición de la que nunca se llegan a despojar las partes involucradas, por lo tanto siempre permanece de forma latente.

Ocupa tiempo y espacio por una serie de elementos que lo constituyen en diversos escenarios, es considerado como una lucha en la que perciben objetivos incompatibles, recompensas escasas e interferencias del otro en realizar sus metas (Wilmot \& Hocker, 2013). Redorta (2004) señala que en la justicia alternativa el conflicto entre las personas involucradas puede llegar asumirse desde cualquiera de los quince perfiles que ha detectado (de recursos escasos, de poder, de valore, estructurales, de identidad, normativos, expectativas, inadaptación, de información, intereses, atributivos, relaciones personales, de inhibición, de legitimación).

El conflicto no surge como una característica propia de la naturaleza genética humana, sino que es el resultado de un error en el desarrollo de las relaciones, de la evolución como personas (Vinyamata, 2002).

El conflicto es un proceso en crecimiento que parte de un estado de necesidad e intereses que se configura en un proceso de crecimiento constante, como se plasma en el siguiente gráfico.

Esquema 1. Proceso del conflicto.

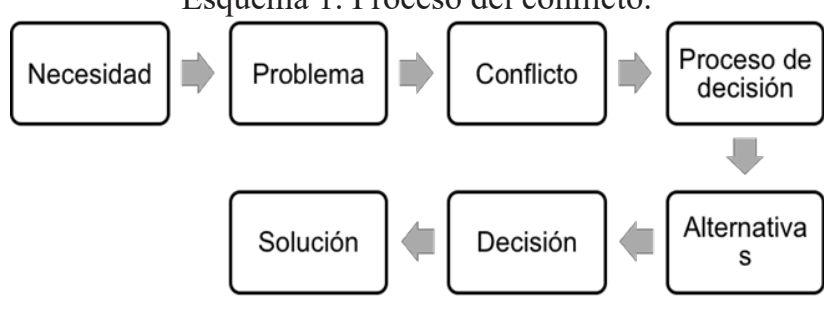

Fuente: Elaboración del autor.

Entelman (2009) se basa en Weber para adjudicar el tejido social en donde se manifiesta la relación social como el comportamiento recíproco de dos o más individuos que orientan, comprenden y resuelven sus conductas teniendo en cuenta las de los otros, con lo que dan sentido a sus actos.

Existe también la distinción del conflicto como formas de transición, evolutivo y transformativo (Galtung, 1978) que puede tener como resultado la propia violencia, maneja la paz y concordia entre las personas, reitera la búsqueda de soluciones que involucre desde la posición individual de la persona hasta su colectivo (Galtung, 2001) supone ver el conflicto como un reto, la incompatibilidad de metas es un gran desafío para las partes involucradas, tanto intelectual como emocional; divide los conflictos para su estudio en tres niveles, micro, y macro nivel.

Lederach (2003) diferencia elementos del conflicto vinculados a las personas implicadas de los elementos propios del proceso, seguido por el conflicto y del problema que subyace a él, por lo que en principio refiere que los elementos del conflicto (Lederach, 1995) se constituyen de acuerdo a los siguientes: las personas (partes involucradas), el poder, la identidad, la comunicación, las percepciones del conflicto, emociones y sentimientos, las posiciones, intereses y necesidades, los valores y los principios y finalmente el campo de las tensiones.

Finalmente, para indagar la solución de un conflicto es necesario identificar sus elementos y causas generadoras, para que su colaboración en la búsqueda de la gestión o posible solución sea la más conveniente, debiéndose llevar a cabo mediante un proceso de mecanismo auto compositivo como la negociación, la mediación, la conciliación y el arbitraje como mecanismos de acceso a la justicia que contribuyen la administración de la propia justicia y más allá de ella.

\section{DISCUSIÓN}

La convivencia es el eje central para poder mantener relaciones pacíficas entre las personas en 
cualquier contexto; por ello desde la institución social de la familia se debe buscar el diálogo y la comprensión mutua que otorgue un clima de confianza. Así el proceso de todo individuo desde la niñez, adolescencia y edad adulta prolifera a crear ciudadanos constructivos y colaborativos con visión transformativa y propositiva del conflicto, siendo el arma principal el diálogo.

Así al momento de estar frente a una controversia no se tornará en polarizaciones del conflicto ni violencia; pero regularmente estas acciones remiten a que se judicialice el conflicto pudiendo prever la gestión del mismo mediante un proceso transformativo como lo son los mecanismos alternativos de negociación, mediación, conciliación y/o arbitraje.

Por último, el conflicto otorga una pauta trascendental para que las controversias sean abordadas mediante procesos educativos, es decir, educar a la ciudadanía para la paz, que asuman activamente sus responsabilidades; preventivas, en la que los ciudadanos actúen o respondan a actos de proliferación de los mecanismos; resolutivas, a partir de lograr o alcanzar acuerdos que ponen fin a los conflictos creando soluciones.

\section{REFERENCIAS}

1. Alcaraz, J. C. \& Lorenzo, J. (2016). El mediador en conflicto. México: Tébar flores.

2. Castillo, C. \& Bautista, M. (2018). Acceso a la Justicia Alternativa: Un Reto Complejo. Utopía y Praxis Latinoamericana, 23 (2),163-176. https://doi.org/10.5281/zenodo.1802160

3. Calvo, M. (2009). Hacía una perspectiva socia jurídica de la teoría positivista del derecho. Novum Jus, 3 (1), 7-34.

4. Fuquen, M. E. (2003). Los conflictos y las formas alternativas de resolución. Tabula Rasa, (1), 265-278. https://doi.org/10.25058/20112742.198

5. Galtung, J. (1978). Conflict as a way of life. Essays in Peace Research, vol. III. Christian Ejleres: Copenhage.

6. Galtung, J. (2001). La resolución de conflictos exige creatividad. España: Universitat de Alicante.

7. Ibáñez, J. (2002). Sobre la metodología cualitativa. Revista Española de Salud Pública, 76(5), 373-380

8. Lederach, J. P. \& Chupp, M. (1995). Conflicto y Violencia. Semilla, Guatemala. Manual de Conciliación. Colombia: Ediciones Clara.

9. Lederach, J. P. (2003). Desafíos y alternativas en la construcción de la paz. Red L@ Revista II (4).

10. Mondragón, F. (2012). "Justicia alternativa en materias civil, mercantil y familiar", en Ferrer, Eduardo (comp.), Procesalismo científico. Tendencias contemporáneas, México: UNAM-Instituto de Investigaciones Jurídicas.

11. Nava, W. (2017). Mecanismos alternativos de resolución de conflictos: un acceso a la justicia consagrado como derecho humano en la Constitución mexicana. Revista Mexicana de Derecho Constitucional. (37), 203-228. http://dx.doi.org/10.22201/iij.24484881e.2017.37.11457

12. Redorta, J. (2004). Cómo analizar los conflictos. La tipología de conflictos como herramienta de mediación. Barcelona: Paidós.

13. Remo, E. (2009). Teoría del conflicto, hacia un nuevo paradigma. España: Gedisa.

14. Silva, G. (2008). La teoría sociológica del conflicto. Un marco teórico necesario. Prolegómenos. Derechos y Valores, 11 (22), 29-43. https://doi.org/10.18359/prole.2506

15. Vinyamata, E. (2002). Manual de prevención y resolución de conflictos. Conciliación, mediación, negociación. Barcelona: Ariel. 